\title{
Analytical and Numerical Study of the Reflection/Transmission Coefficients in Slight Viscoelastic Medium
}

\author{
Fateh Bouchaala ${ }^{1}$, Claude Guennou ${ }^{2}$ \\ ${ }^{1}$ Petroleum Institute of Abu-Dhabi, Abu Dhabi, UAE \\ ${ }^{2}$ Domaines Oceaniques Laboratory, Brest, France \\ Email: fbouchaala@pi.ac.ae
}

Received 29 March 2014; revised 25 April 2014; accepted 18 May 2014

Copyright @ 2014 by authors and Scientific Research Publishing Inc.

This work is licensed under the Creative Commons Attribution International License (CC BY). http://creativecommons.org/licenses/by/4.0/

(c) (i) Open Access

\section{Abstract}

The study done in this paper brings out the effect of the viscoelasticity on the reflection/transmission coefficients. The knowledge of this effect can be useful for several applications, such as enhancing the resolution of the seismic sections, fluid and fracture detection. It can also have other applications different from the geophysical domain, as the study of the bonding between the materials in the civil engineering domain. We use the complex Lame coefficients in the continuity equations at the boundary layers to get the analytical expressions of the reflection/transmission coefficients in viscoelastic media. The coefficients can be divided into two parts, the first part is independent from the quality factor, and it corresponds to the elastic reflection/transmission coefficients. The second part is dependent on the quality factor contrast and it represents the contribution of the viscoelasticity on the reflection/transmission coefficients. From the numerical study it appears that the effect of the viscoelasticity is significant near to the critical angles. This effect is not clear and it is difficult to interpret and we do not know if it has a physical meaning or it is only a mathematical artifact that is why it is better to be far from the critical angles for seismic investigation.

\section{Keywords}

Viscoelasticity, Quality Factor, Reflection/Transmission

\section{Introduction}

The estimation of the reflection/transmission coefficients of the seismic waves is paramount for the investigation

How to cite this paper: Bouchaala, F. and Guennou, C. (2014) Analytical and Numerical Study of the Reflection/Transmission Coefficients in Slight Viscoelastic Medium. International Journal of Geosciences, 5, 908-924. 
of the natural medium and to know their geometrical proprieties. In a perfect elastic medium, these coefficients are computed from the impedance contrast between two neighbouring layers [1]-[3]. In the viscoelastic media in addition to the impedance contrast, the viscoelasticity contrast between the two neighbouring layers should be taken into account. Usually the viscoelasticity of the medium is quantified by using the quality factor $Q$, which is inversely proportional to the energy loss $\left(\frac{\Delta E}{E}\right)$ of the seismic wave:

$$
\frac{1}{Q}=\frac{\Delta E}{2 \pi E}
$$

Buchen [4] has studied the propagation of the seismic wave $S H$ in a layered and slight viscoelastic media. He used complex wave number to compute the reflection/transmission coefficients; the imaginary part of the wave number takes account the viscoelastic functionality. He noted that the difference between the reflection/transmission coefficients in the elastic and viscoelastic media is important when the quality factor contrast between two neighbouring layers is significant. Krebes [5], has done the same study as Buchen [4], but he considered the medium was highly viscoelastic. He noted that even by considering the medium highly viscoelastic, the equality of the quality factor between two neighbouring layers implies the independence of the reflection/transmission coefficients from the viscosity of the medium. The results of Buchen and Krebes, were confirmed by Carcione [6], who studied also the effect of the anisotropy on the reflection/transmission coefficients.

In this paper, first we do an analytical study of the reflection/transmission coefficients in the slight viscoelastic medium. From this study we provide the analytical expressions of these coefficients for all possible incident wave ( $S H, P$ and $S V$ ), which allows to see clearly the contribution of the viscoelasticity on the reflection/ transmission coefficients. This analytical study is followed by a numerical example; through it we apply the analytical expressions on synthetic data. So far no study about the reflection/transmission coefficients in slight viscoelastic media has been more complete than this one.

\section{Analytical Study}

Reflection/transmission of plane seismic waves is a local phenomenon, involving local physical proprieties of two medium (1) and (2) at the discontinuity point $I$. Locally, in the vicinity of the point $I$, we can consider that the interface is plane and having normal $N$ (Figure 1). At the point $I$ the seismic energy can be divided on reflected and transmitted, and it can generate two waves: reflected and transmitted.

An incident $P$ wave as an incident $S V$ wave can generate reflected $P$ or $S V$ wave and transmitted $P$ or $S V$ wave. However, an incident $S H$ wave can generates only reflected or transmitted $S H$ wave. The reflected or transmitted waves which are different from the incident wave, they are called converted waves, such $P$-SV reflection for example.

In this study the incident wave is indicated by the subscript $i$, the reflected wave by the subscript $r$ and the transmitted wave by the subscript $t$. The viscoelastic reflection/transmission coefficient are designed by the variables $R_{V} / T_{V}$.

In this section we put just the main equations leading to the $R_{V} / T_{V}$ coefficients. More details are given in the thesis of Bouchaala [7].

\section{Displacement Continuity}

The displacement of the wave particles at the instant $t$ and the position $\boldsymbol{x}$ can be expressed as [8],

$$
\boldsymbol{u}(\boldsymbol{x}, t)=S(\omega) \boldsymbol{U}(\boldsymbol{x}) \exp (-i \omega(t-\boldsymbol{p} \boldsymbol{x}))
$$

where $S(\omega)$ is the source term, $\omega$ is the circular frequency, $\boldsymbol{U}(\boldsymbol{x})$ is the amplitude vector at the position $\boldsymbol{x}$. In the orthonormal basis $\left(\boldsymbol{t}, \boldsymbol{n}_{1}, \boldsymbol{n}_{2}\right)$ (Figure 2) the amplitude vector $\boldsymbol{U}$ has the components $(A, B, C)$ and can be written as,

$$
\boldsymbol{U}(\boldsymbol{x})=A \boldsymbol{t}+B \boldsymbol{n}_{1}+C \boldsymbol{n}_{2}
$$

$\boldsymbol{p}$ is the slowness, which can be computed from the velocity $V$, the propagation direction $\boldsymbol{t}$ and the quality factor $Q$ by: 


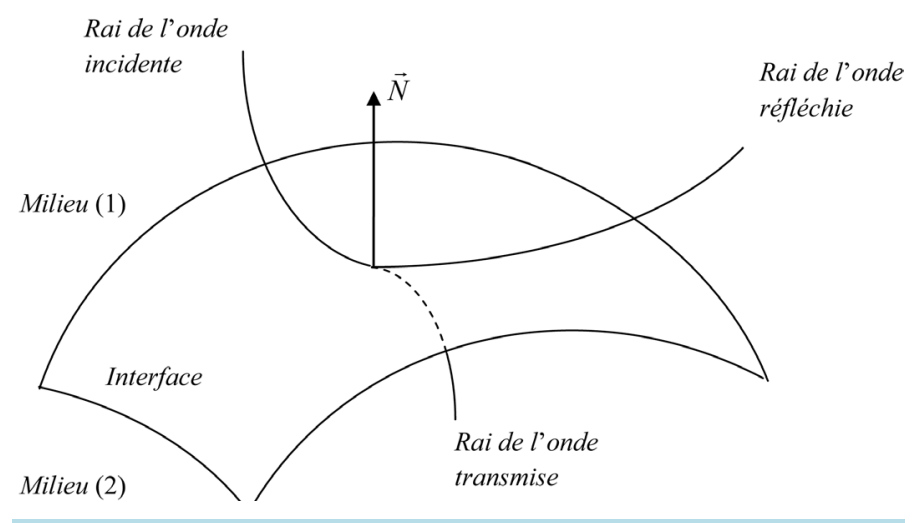

Figure 1. Incident, reflected and transmitted waves in a medium composed from two layers (1) and (2), $I$ is the intersection point between the incident wave and the interface, $\boldsymbol{N}$ is the unit vector normal to the interface at the point $I$.

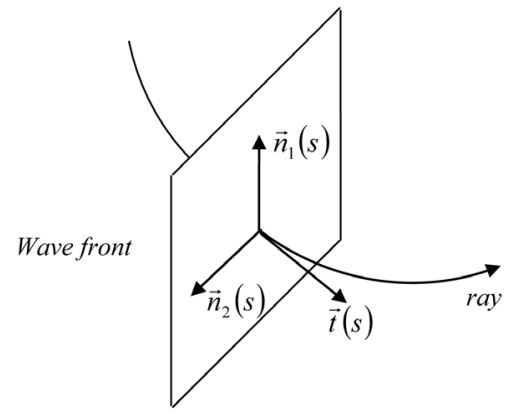

Figure 2. The direct orthonormal basis $\left(\boldsymbol{t}(s), \boldsymbol{n}_{1}(s), \boldsymbol{n}_{2}(s)\right)$. The unit vector $\boldsymbol{t}(s)$ is the first Fresnel vector, tangent to the ray, the unit vectors, $\boldsymbol{n}_{1}(s)$ and $\boldsymbol{n}_{2}(s)$ are orthogonal between themselves.

$$
\boldsymbol{p}=\frac{\boldsymbol{t}}{V}\left(1-\frac{i}{2 Q}\right)
$$

In the elastic case the slowness vector is real and independent form $Q, \quad \boldsymbol{p}=\frac{\boldsymbol{t}}{V}$.

The continuity of the displacement vector at the point $I$ (Figure 3) is expressed as:

$$
[\boldsymbol{u}(I, t)]_{1}=[\boldsymbol{u}(I, t)]_{2}
$$

The displacement at the point $I$ in the medium (1) is due to the incident and reflected waves:

$$
[\boldsymbol{u}(I, t)]_{1}=S(\omega)\left(\boldsymbol{U}_{i}+\boldsymbol{U}_{r}\right) \exp (-i \omega(t-\boldsymbol{p} \boldsymbol{x}(I)))
$$

The displacement at the point $I$ in the medium (2) is due only to the transmitted wave:

$$
[\boldsymbol{u}(I, t)]_{2}=S(\omega) \boldsymbol{U}_{t} \exp (-i \omega(t-\boldsymbol{p} \boldsymbol{x}(I)))
$$

We use the expressions of the displacement given in the Equations (6) and (7) in the continuity Equation (5) and we obtain:

$$
\boldsymbol{U}_{i}+\boldsymbol{U}_{r}=\boldsymbol{U}_{t}
$$

The projection of the Equation (8) on the unit vector $\boldsymbol{X}_{2}$ (Figure 3) gives:

$$
B_{i}+B_{r}=B_{t}
$$




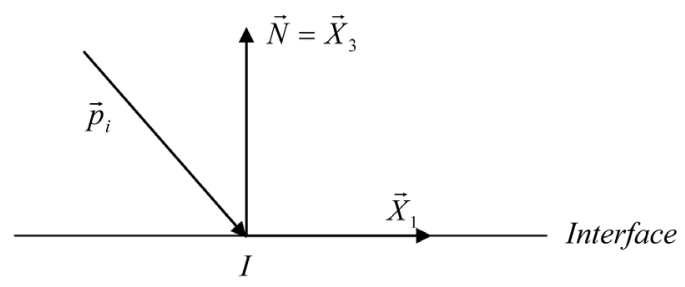

Figure 3. The direct orthonormal basis $\left(\boldsymbol{X}_{1}, \boldsymbol{X}_{2}, \boldsymbol{X}_{3}\right)$,

the vector $\boldsymbol{X}_{2}=\boldsymbol{n}$ is perpendicular to the figure.

And in the incident plane $\left(\boldsymbol{X}_{1}, \boldsymbol{X}_{3}\right)$ (Figure 3) it gives:

$$
A_{i} \boldsymbol{t}_{i}+C \boldsymbol{n}_{2 i}+A_{r} \boldsymbol{t}_{r}+C_{r} \boldsymbol{n}_{2 r}=A_{t} \boldsymbol{t}_{t}+C_{t} \boldsymbol{n}_{2 t}
$$

\section{Stress Continuity}

The continuity condition of stress vector at the point $I$, is expressed by the following equation:

$$
[\overline{\bar{\sigma}}(I, t) \boldsymbol{N}]_{1}=[\overline{\bar{\sigma}}(I, t) \boldsymbol{N}]_{2}
$$

where $[\overline{\bar{\sigma}}(I, t) N]_{1}$ is the stress vector at the point $I$, generated by the incident and the reflected waves (propagating in the medium (1)), and applied on the elementary surface having a normal vector $N$.

$[\overline{\bar{\sigma}}(I, t) N]_{2}$ is the stress vector at the point $I$ generated by the transmitted wave (propagating in the medium (2)), and applied on the elementary surface having a normal vector $\boldsymbol{N}$.

In the isotropic media, the generalized Hook's law can be simplified as:

$$
\overline{\bar{\sigma}}=\lambda(\nabla \cdot \boldsymbol{u}) \overline{\bar{I}}+\mu\left(\overline{\bar{\nabla}} \mathbf{u}+\nabla^{t} \mathbf{u}\right)
$$

where $\lambda$ and $\mu$ are the complex Lame coefficients, their imaginary parts contain the viscoelastic functionality of the medium. In the elastic case these coefficients are real.

The projection of the Equation (12) in the orthonormal basis $\left(\boldsymbol{X}_{1}, \boldsymbol{X}_{2}, \boldsymbol{X}_{3}\right)$ (Figure 3), leads to the following expression of stress vector $\overline{\bar{\sigma}} \boldsymbol{N}=\overline{\bar{\sigma}} \boldsymbol{X}_{3}$ :

$$
\overline{\bar{\sigma}} \boldsymbol{X}_{3}=\mu\left(\frac{\partial u_{1}}{\partial X_{3}}+a u_{3}\right) \boldsymbol{X}_{1}+\mu \frac{\partial u_{2}}{\partial X_{3}} \boldsymbol{X}_{2}+\left[\lambda\left(a u_{1}+\frac{\partial u_{3}}{\partial X_{3}}\right)+2 \mu \frac{\partial u_{3}}{\partial X_{3}}\right] \boldsymbol{X}_{3}
$$

where

$$
a=\frac{\sin \left(\theta_{i}\right)}{V_{i}}\left(1+\frac{i}{2 Q}\right), \quad \theta_{i} \text { is the incident angle }
$$

So, by using the Equation (14), the continuity condition (12) in theorthonormal basis $\left(\boldsymbol{X}_{1}, \boldsymbol{X}_{2}, \boldsymbol{X}_{3}\right)$, we get a system of three equations:

$$
\begin{aligned}
& {\left[\mu\left(\frac{\partial u_{1}}{\partial X_{3}}+a u_{3}\right)\right]_{1}=\left[\mu\left(\frac{\partial u_{1}}{\partial X_{3}}+a u_{3}\right)\right]_{2}} \\
& {\left[\mu \frac{\partial u_{2}}{\partial X_{3}}\right]_{1}=\left[\mu \frac{\partial u_{2}}{\partial X_{3}}\right]_{2}} \\
& {\left[\lambda\left(a u_{1}+\frac{\partial u_{3}}{\partial X_{3}}\right)+2 \mu \frac{\partial u_{3}}{\partial X_{3}}\right]_{1}=\left[\lambda\left(a u_{1}+\frac{\partial u_{3}}{\partial X_{3}}\right)+2 \mu \frac{\partial u_{3}}{\partial X_{3}}\right]_{2}}
\end{aligned}
$$

From the Equation (2) the derivation of the components of the vector displacement can be given as,

$$
\frac{\partial u_{i}}{\partial X_{j}}=i \omega\left(\boldsymbol{p} \cdot \boldsymbol{X}_{j}\right) u_{i}
$$


The system of Equation (15) contains one equation involving only the second component $\left(u_{2}\right)$, corresponding to the displacement of the wave $S H$ and also two other equations, involving the first and the third components, $u_{1}$ and $u_{3}$, corresponding to the displacement of $P$ and $S V$ waves, respectively. This means, that the reflection/transmission problem can be divided in two sub-problems: $P$-SV and $S H$.

$$
\begin{gathered}
\left(\begin{array}{l}
A_{r} \\
B_{r} \\
C_{r}
\end{array}\right)=\boldsymbol{R}\left(\begin{array}{l}
A_{i} \\
B_{i} \\
C_{i}
\end{array}\right)=\left(\begin{array}{ccc}
R_{V}^{P \rightarrow P} & 0 & R_{V}^{S V \rightarrow P} \\
0 & R_{D}^{S H \rightarrow S H} & 0 \\
R_{V}^{P \rightarrow S V} & 0 & R_{V}^{S V \rightarrow S V}
\end{array}\right)\left(\begin{array}{l}
A_{i} \\
B_{i} \\
C_{i}
\end{array}\right) \\
\left(\begin{array}{l}
A_{t} \\
B_{t} \\
C_{t}
\end{array}\right)=\boldsymbol{T}\left(\begin{array}{l}
A_{i} \\
B_{i} \\
C_{i}
\end{array}\right)=\left(\begin{array}{ccc}
T_{V}^{P \rightarrow P} & 0 & T_{V}^{S V \rightarrow P} \\
0 & T_{D}^{S H \rightarrow S H} & 0 \\
T_{V}^{P \rightarrow S V} & 0 & T_{V}^{S V \rightarrow S V}
\end{array}\right)\left(\begin{array}{l}
A_{i} \\
B_{i} \\
C_{i}
\end{array}\right)
\end{gathered}
$$

$\boldsymbol{R}$ and $\boldsymbol{T}$ indicate the matrix of the reflection and transmission coefficients, respectively.

We use the derivation in the Equation (16) to get the projection in the orthonormal basis $\left(\boldsymbol{X}_{1}, \boldsymbol{X}_{2}, \boldsymbol{X}_{3}\right)$ of displacement and stress vectors due to the incident, reflected and transmitted waves of $S H, S V$ and $P$ waves, which are given in the Tables 1-4.

Table 1. Component in the basis $\left(\boldsymbol{X}_{1}, \boldsymbol{X}_{2}, \boldsymbol{X}_{3}\right)$ of displacement vectors at the point $I$ for incident, reflected and transmitted waves (SH Reflection/Transmission).

\begin{tabular}{ll}
\hline $\begin{array}{l}\text { Displacement vector at point } I \text { Of the incident wave } S H \\
\times S(\omega) \exp (-i \omega(t-T(I)))\end{array}$ & $\begin{array}{l}\text { Displacement vector at point } I \text { Of the generated waves } \\
\times S(\omega) \exp (-i \omega(t-T(I)))\end{array}$ \\
\hline$\left(0, B_{i}, 0\right)$ & $\begin{array}{l}\text { Reflected } S H\left(0, B_{r}, 0\right) \\
\text { Transmitted } S H\left(0, B_{t}, 0\right)\end{array}$ \\
\hline
\end{tabular}

Table 2. Component in the basis $\left(\boldsymbol{X}_{1}, \boldsymbol{X}_{2}, \boldsymbol{X}_{3}\right)$ of the stress vector $\overline{\bar{\sigma}} \boldsymbol{X}_{3}$ at point $I$ Due to incident, reflected and transmitted waves (Reflection/Transmission $S H$ ).

\begin{tabular}{ll}
\hline $\begin{array}{l}\text { Stress vector at point } I \text { due to incident wave } S H \\
\times i \omega S(\omega) \exp (-i \omega(t-T(I)))\end{array}$ & $\begin{array}{l}\text { Stress vector at point } I \text { due to generated waves } \\
\times i \omega S(\omega) \exp (-i \omega(t-T(I)))\end{array}$ \\
\hline$-\frac{\mu_{1}^{*}}{\beta_{1}^{*}}\left(1-a^{* 2} \beta_{1}^{* 2}\right)^{1 / 2}\left(0, B_{i}, 0\right)$ & Reflected $-\frac{\mu_{1}^{*}}{\beta_{1}^{*}}\left(1-a^{* 2} \beta_{1}^{* 2}\right)^{1 / 2}\left(0, B_{i}, 0\right) \frac{\mu_{1}^{*}}{\beta_{1}^{*}}\left(1-a^{* 2} \beta_{1}^{* 2}\right)^{1 / 2}\left(0, B_{r}, 0\right)$ \\
& Transmitted $-\frac{\mu_{2}^{*}}{\beta_{2}^{*}}\left(1-a^{* 2} \beta_{2}^{* 2}\right)^{1 / 2}\left(0, B_{t}, 0\right)$ \\
\hline
\end{tabular}

Table 3. Component in the basis $\left(\boldsymbol{X}_{1}, \boldsymbol{X}_{2}, \boldsymbol{X}_{3}\right)$ of the displacement vector at point $I$ for incident, reflected and transmitted waves (Reflection/Transmission $P$-SV).

\begin{tabular}{ll}
\hline $\begin{array}{l}\text { Displacement vector at point } I \text { Of incident waves } \\
\times S(\omega) \exp (-i \omega(t-T(I)))\end{array}$ & $\begin{array}{l}\text { Displacement vector at point } I \text { of generated waves } \\
\times S(\omega) \exp (-i \omega(t-T(I)))\end{array}$ \\
\hline Reflected $P A_{t}\left(a^{*} \alpha_{1}^{*}, 0, P_{1}^{*}\right)$ \\
Incidente $P \quad A_{t}\left(a^{*} \alpha_{1}^{*}, 0,-P_{1}^{*}\right)$ & Transmitted $P \quad A_{t}\left(a^{*} \alpha_{2}^{*}, 0,-P_{3}^{*}\right)$ \\
& Reflected $S V C_{r}\left(-P_{2}^{*}, 0, a^{*} \beta_{1}^{*}\right)$ \\
& Transmitted $S V C_{t}\left(P_{4}^{*}, 0, a^{*} \beta_{2}^{*}\right)$ \\
& Reflected $P A_{r}\left(a^{*} \alpha_{1}^{*}, 0, P_{1}^{*}\right)$ \\
& Transmitted $P \quad A_{t}\left(a^{*} \alpha_{2}^{*}, 0,-P_{3}^{*}\right)$ \\
& Reflected $S V C_{r}\left(-P_{2}^{*}, 0, a^{*} \beta_{1}^{*}\right)$ \\
& Transmitted $S V C_{t}\left(P_{4}^{*}, 0, a^{*} \beta_{2}^{*}\right)$ \\
\hline
\end{tabular}


Table 4. Component in the basis $\left(\boldsymbol{X}_{1}, \boldsymbol{X}_{2}, \boldsymbol{X}_{3}\right)$ of stress vector at $I$ due to incident, reflected, and transmitted waves (Reflection/Transmission P-SV).

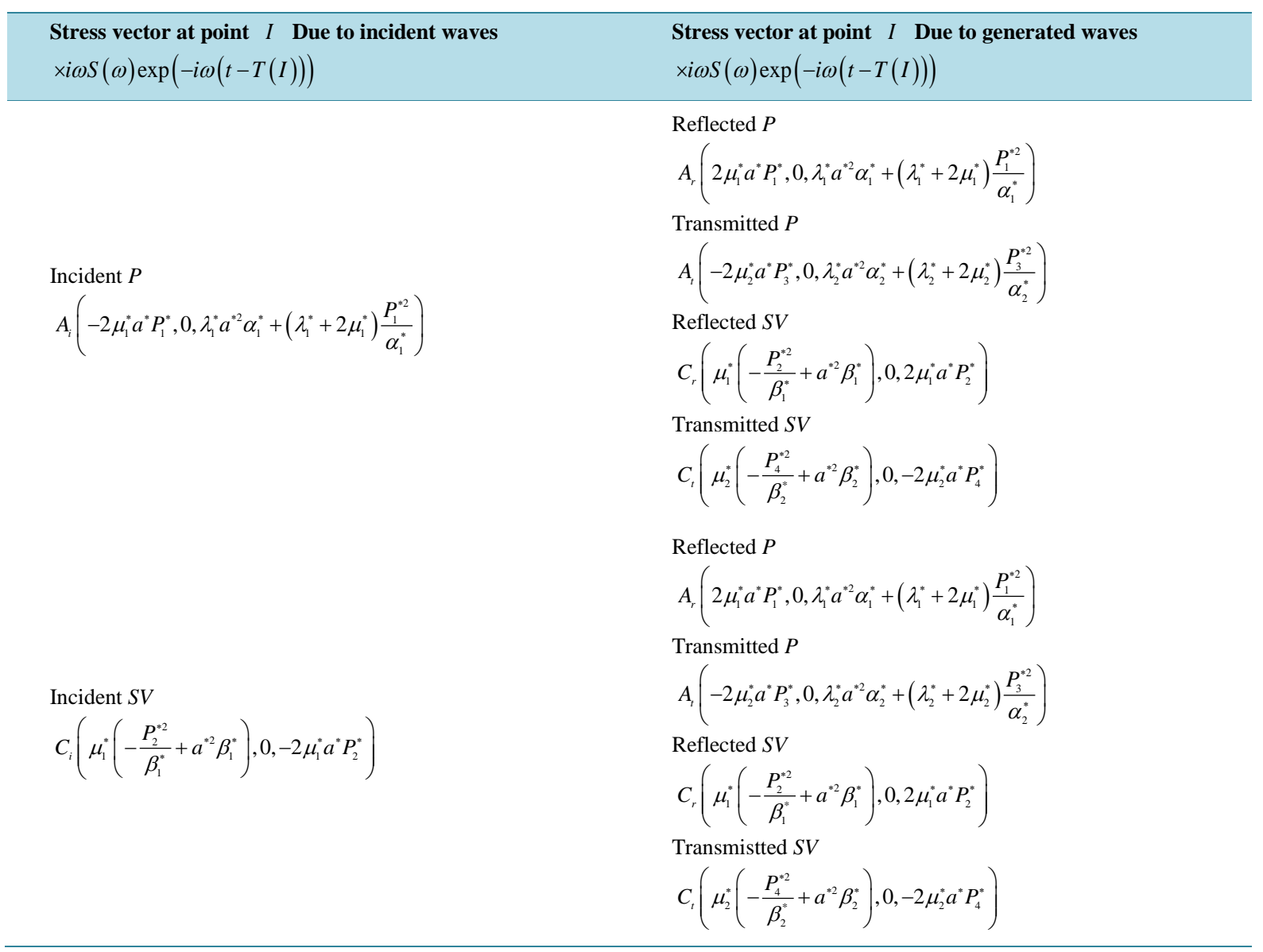

Before starting our analytical development, we remind the following results:

$$
\mu=\rho \beta^{*}, \quad \lambda=\rho\left(\alpha^{* 2}-2 \beta^{* 2}\right)
$$

where $\beta^{*}$ and $\alpha^{*}$ are the complex $S$ and $P$ wave velocities which can be expressed from the real velocities $\beta$ and $\alpha$ and the quality factors $Q_{P}$ and $Q_{S}$ (see Appendix).

\section{SH Problem}

We assume $S H$ incident wave, with the amplitude vector, $\boldsymbol{U}_{i}=B_{i} \boldsymbol{n}_{1}=B_{i} \boldsymbol{X}_{2}$. The continuity Equations (9) and (15) at the interface show that the incident $S H$ wave, generates only $S H$ waves, reflected or transmitted.

After using the projection of the stress vector in the basis $\left(\boldsymbol{X}_{1}, \boldsymbol{X}_{2}, \boldsymbol{X}_{3}\right)$ given in the Table 2 and replacing $\mu$ by its expression in Equation (19), the Equation (15) becomes,

$$
\left(B_{i}-B_{r}\right) \rho_{1} \beta_{1}^{*} P_{2}^{*}=B_{t} \rho_{2} \beta_{2}^{*} P_{4}^{*}
$$

After using the Equation (9), the Equation (20) can be written as,

$$
\left(B_{i}-B_{r}\right) \rho_{1} \beta_{1}^{*} P_{2}^{*}=\left(\beta_{i}+\beta_{r}\right) \rho_{2} \beta_{2}^{*} P_{4}^{*}
$$

or

$$
\left(2 B_{i}-B_{t}\right) \rho_{1} \beta_{1}^{*} P_{2}^{*}=\beta_{t} \rho_{2} \beta_{2}^{*} P_{4}^{*}
$$

The $R_{V} / T_{V}$ coefficients are defined as, 


$$
R_{V}^{S H \rightarrow S H}=\frac{B_{r}}{B_{i}}, \quad T_{V}^{S H \rightarrow S H}=\frac{B_{t}}{B_{i}}
$$

After dividing the Equations (21) and (22) by $B_{i}$, we obtain expression of $R_{V} / T_{V}$ coefficients

$$
\begin{gathered}
R_{V}^{S H \rightarrow S H}=\frac{\rho_{1} \beta_{1}^{*} P_{2}^{*}-\rho_{2} \beta_{2}^{*} P_{4}^{*}}{\rho_{1} \beta_{1}^{*} P_{2}^{*}+\rho_{2} \beta_{2}^{*} P_{4}^{*}} \\
T_{V}^{S H \rightarrow S H}=\frac{B_{t}}{B_{i}}=\frac{2 \rho_{1} \beta_{1}^{*} P_{2}^{*}}{\rho_{1} \beta_{1}^{*} P_{2}^{*}+\rho_{2} \beta_{2}^{*} P_{4}^{*}}
\end{gathered}
$$

We replace the parameters indicated by an asterisk in Equations (24) and (25) by their expressions shown in the Appendix, to obtain the final expressions of the $R_{V} / T_{V}$ coefficients,

$$
\begin{aligned}
& R_{V}^{S H \rightarrow S H}=R_{E}^{S H \rightarrow S H}-2 i \frac{\rho_{1} \rho_{2} \beta_{1} \beta_{2} P_{2} P_{4}}{\left(\rho_{1} \beta_{1} P_{2}+\rho_{2} \beta_{2} P_{4}\right)^{2}}\left(\frac{a^{2} \beta_{2}^{2}}{2 P_{4}^{2}}-1\right)\left(\frac{1}{Q_{S 2}}-\frac{1}{Q_{S 1}}\right) \\
& T_{V}^{S H \rightarrow S H}=T_{E}^{S H \rightarrow S H}-2 i \frac{\rho_{1} \rho_{2} \beta_{1} \beta_{2} P_{2} P_{4}}{\left(\rho_{1} \beta_{1} P_{2}+\rho_{2} \beta_{2} P_{4}\right)^{2}}\left(\frac{a^{2} \beta_{2}^{2}}{2 P_{4}^{2}}-1\right)\left(\frac{1}{Q_{S 2}}-\frac{1}{Q_{S 1}}\right)
\end{aligned}
$$

where $P_{2}=\left(1-\sin \theta_{i}\right)^{1 / 2}$ and $P_{4}=\left(1-\frac{\beta_{2}}{\beta_{1}} \sin \theta_{i}\right)^{1 / 2}$.

The terms $R_{E}^{S H \rightarrow S H}$ and $R_{E}^{S H \rightarrow S H}$ in the Equations (26) and (27), corresponds respectively to the reflection and transmission coefficients of the elastic media which are dependent on the impedance contrast $\rho \beta$ between the two media. The second term of the equations is due to the viscosity effect on the $R_{V} / T_{V}$ coefficients, it is proportional to the quality factor contrast, $\frac{1}{Q_{S_{2}}}-\frac{1}{Q_{S_{1}}}$ between the two media. This means that with a zero contrast in the quality factor, the $R_{V} / T_{V}$ coefficients are similar to those of the elastic case.

\section{P-SV Problem}

$P$ and $S V$ incident waves can generate reflected or transmitted $P$ and $S V$ waves. After using the projection of the displacement and stress vector in the basis $\left(\boldsymbol{X}_{1}, \boldsymbol{X}_{2}, \boldsymbol{X}_{3}\right)$ given in the Table 3 and Table 4, we replace $\lambda$ and $\mu$ by their expressions in Equation (19), thecontinuity Equations (9) and (15) can be written respectively as,

For $P$ incident wave

$$
\begin{gathered}
a \alpha_{1}^{*}\left(A_{i}+A_{r}\right)-P_{2}^{*} C_{r}=a \alpha_{2}^{*} A_{t}+P_{4}^{*} C_{t} \\
P_{1}^{*}\left(A_{i}-A_{r}\right)-a \beta_{1}^{*} C_{r}=P_{3}^{*} A_{t}-a \beta_{2}^{*} C_{t} \\
2 \rho_{1} \beta_{1}^{* 2} a P_{1}^{*}\left(A_{i}-A_{r}\right)+\rho_{1} \beta_{1}^{*}\left(1-2 a^{2} \beta_{1}^{* 2}\right) C_{r}=2 \rho_{2} \beta_{2}^{* 2} a P_{3}^{*} A_{t}+\rho_{2} \beta_{2}^{*}\left(1-2 a^{2} \beta_{2}^{* 2}\right) C_{t} \\
\rho_{1} \alpha_{1}^{*}\left(1-2 a^{2} \beta_{1}^{* 2}\right)\left(A_{i}+A_{r}\right)+2 \rho_{1} \beta_{1}^{* 2} a P_{2}^{*} C_{r}=\rho_{2} \alpha_{2}^{*}\left(1-2 a^{2} \beta_{2}^{* 2}\right) A_{t}-2 \rho_{2} \beta_{2}^{* 2} a P_{4}^{*} C_{t}
\end{gathered}
$$

For $S V$ incident wave,

$$
\begin{gathered}
P_{2}^{*}\left(C_{i}-C_{r}\right)+a \alpha_{1}^{*} A_{r}=a \alpha_{2}^{*} A_{t}+P_{4}^{*} C_{t} \\
a \beta_{1}^{*}\left(C_{i}+C_{r}\right)+P_{1}^{*} A_{r}=-P_{3}^{*} A_{t}+a \beta_{2}^{*} C_{t} \\
\rho_{1} \beta_{1}^{*}\left(1-2 a^{2} \beta_{1}^{* 2}\right)\left(C_{i}+C_{r}\right)-2 \rho_{1} \beta_{1}^{* 2} a P_{1}^{*} A_{r}=2 \rho_{2} \beta_{2}^{* 2} a P_{3}^{*} A_{t}+\rho_{2} \beta_{2}^{*}\left(1-2 a^{2} \beta_{2}^{* 2}\right) C_{t} \\
-2 \rho_{1} \beta_{1}^{* 2} a P_{2}^{*}\left(C_{i}-C_{r}\right)+\rho_{1} \alpha_{1}^{*}\left(1-2 a^{2} \beta_{1}^{* 2}\right) A_{r}=\rho_{2} \alpha_{2}^{*}\left(1-2 a^{2} \beta_{2}^{* 2}\right) A_{t}-2 \rho_{2} \beta_{2}^{* 2} a P_{4}^{*} C_{t}
\end{gathered}
$$

The $R_{V} / T_{V}$ coefficients are defined as,

For $P$ incident wave, 


$$
R_{V}^{P \rightarrow P^{*}}=\frac{A_{r}}{A_{i}}, \quad R_{V}^{P \rightarrow S V^{*}}=\frac{C_{r}}{A_{i}}, \quad T_{V}^{P \rightarrow S V^{*}}=\frac{C_{t}}{A_{i}}
$$

For $S$ incident wave,

$$
R_{V}^{S V \rightarrow P^{*}}=\frac{A_{r}}{C_{i}}, \quad R_{V}^{S V \rightarrow S V^{*}}=\frac{C_{r}}{C_{i}}, \quad T_{V}^{S V \rightarrow P^{*}}=\frac{A_{t}}{C_{i}}, \quad T_{V}^{S V \rightarrow S V^{*}}=\frac{C_{t}}{C_{i}}
$$

By combining the Equations (27) to (32), $R_{V} / T_{V}$ coefficients can be expressed in matrix form, For $P$ incident wave,

$$
\left[\begin{array}{c}
R_{V}^{P \rightarrow P} \\
R_{V}^{P \rightarrow S V} \\
T_{V}^{P \rightarrow P} \\
T_{V}^{P \rightarrow S V}
\end{array}\right]=\overline{\bar{M}}^{-1}\left[\begin{array}{c}
a \alpha_{1}^{*} \\
P_{1}^{*} \\
2 \rho_{1} \beta_{1}^{* 2} a P_{1}^{*} \\
\rho_{1} \alpha_{1}^{*}\left(1-2 a^{2} \beta_{1}^{* 2}\right)
\end{array}\right]
$$

For SV incident wave,

$$
\begin{aligned}
& {\left[\begin{array}{c}
R_{D}^{S V \rightarrow P} \\
R_{D}^{S V \rightarrow S V} \\
T_{D}^{S V \rightarrow P} \\
T_{D}^{S V \rightarrow S V}
\end{array}\right]=\overline{\bar{M}}^{-1}\left[\begin{array}{c}
P_{2}^{*} \\
-a \beta_{1}^{*} \\
\rho_{1} \beta_{1}^{*}\left(1-2 a^{2} \beta_{1}^{* 2}\right) \\
-2 \rho_{1} \beta_{1}^{* 2} a P_{2}^{*}
\end{array}\right]} \\
& \overline{\overline{\boldsymbol{M}}}=\left[\begin{array}{cccc}
-a \alpha_{1}^{*} & P_{2}^{*} & a \alpha_{2}^{*} & P_{4}^{*} \\
P_{1}^{*} & a \beta_{1}^{*} & P_{3}^{*} & -a \beta_{2}^{*} \\
2 \rho_{1} \beta_{1}^{* 2} a P_{1}^{*} & -\rho_{1} \beta_{1}^{*}\left(1-2 a^{2} \beta_{1}^{* 2}\right) & 2 \rho_{2} \beta_{2}^{* 2} a P_{3}^{*} & \rho_{2} \beta_{2}^{*}\left(1-2 a^{2} \beta_{2}^{* 2}\right) \\
-\rho_{1} \alpha_{1}^{*}\left(1-2 a^{2} \beta_{1}^{* 2}\right) & -2 \rho_{1} \beta_{1}^{* 2} a P_{2}^{*} & \rho_{2} \alpha_{2}^{*}\left(1-2 a^{2} \beta_{2}^{* 2}\right) & -2 \rho_{2} \beta_{2}^{* 2} a P_{4}^{*}
\end{array}\right]
\end{aligned}
$$

We invert the matrix $\overline{\bar{M}}$ in the Equations (33) and (34), then we obtain the analytical expressions of all possible reflection and transmission coefficients from $P$ and $S V$ incident waves, under the assumption that $Q_{P}=Q_{S}$ :

$$
\begin{aligned}
& R_{V}^{P \rightarrow P}=R_{E}^{P \rightarrow P}+i r^{P \rightarrow S V}\left(\frac{1}{Q_{2}}-\frac{1}{Q_{1}}\right) \quad R_{V}^{P \rightarrow S V}=R_{E}^{P \rightarrow S V}+i r^{P \rightarrow S V}\left(\frac{1}{Q_{2}}-\frac{1}{Q_{1}}\right) \\
& T_{V}^{P \rightarrow P}=T_{E}^{P \rightarrow P}+i t^{P \rightarrow P}\left(\frac{1}{Q_{2}}-\frac{1}{Q_{1}}\right) \quad T_{V}^{P \rightarrow S V}=T_{E}^{P \rightarrow S V}+i t^{P \rightarrow S V}\left(\frac{1}{Q_{2}}-\frac{1}{Q_{1}}\right) \\
& R_{V}^{S V \rightarrow P}=R_{E}^{S V \rightarrow P}+i r^{S V \rightarrow P}\left(\frac{1}{Q_{2}}-\frac{1}{Q_{1}}\right) \quad T^{S V \rightarrow S V}=T_{E}^{S V \rightarrow S V}+i t^{S V \rightarrow S V}\left(\frac{1}{Q_{2}}-\frac{1}{Q_{1}}\right)
\end{aligned}
$$

Such the $S H$ incident wave case, the above equations can be divided in two terms, the first one corresponds respectively to the reflection and transmission coefficients of the elastic media and the second one corresponds to the contribution of the viscosity. The contribution is proportional to the contrast of the viscosity $\frac{1}{Q_{2}}-\frac{1}{Q_{1}}$, therefore if the quality factor contrast equals zero, we get back at the elastic case.

The exact expressions of the second terms in Equation (36) are given in the Appendix.

\section{Numerical Study}

\section{Synthetic Data}

To have further insight about the effect of the viscoelasticity on the $R_{V} / T_{V}$ coefficients, we apply the above expressions of the coefficients on a synthetic data. We assume two cases, in the first case we assume a low im- 
pedance contrast between the medium (1) and the medium (2), and in the second case we assume a big impedance contrast between the two media (Table 5). The medium (1) in the first case has similar characteristic as a sandstone formation and as marlstone formation in the second case. The medium (2) has similar characteristic as a limestone formation in the two cases [9].

We should respect the slight viscosity assumption, for the choice of the quality factor values $(Q \leq 20)$. For each case and each reflection or transmission, we choose: $Q_{1}=20$ and $Q_{2}=1000$, in the first time then the inverse: $Q_{1}=1000$ and $Q_{2}=20$. The curves of the $R_{V} / T_{V}$ coefficients versus incidence angles for the chosen values of the quality factor, are compared to the elastic case $\left(Q_{1}=Q_{2}\right)$.

\section{Results}

The curves (Figures 4-13) of the $R_{V} / T_{V}$ coefficients from all possible incident waves ( $P$-SV, $S H$ ), show that the viscoelasticity of the medium disturbs there flection/transmission-coefficients around the critical angles (Table 6 and Table 7). The difference between the elastic $R_{V} / T_{V}$ coefficients, indicated by the black color

Table 5. Synthetic data.

\begin{tabular}{ccccc}
\hline Data & \multicolumn{3}{c}{ Medium1 } & \multicolumn{2}{c}{ Medium 2 } \\
\hline Velocity $(\mathrm{km} / \mathrm{s})$ & $V p_{1}$ & $V s_{1}$ & $V s_{2}$ \\
\hline Case 1 & 3.50 & 2.02 & 5.00 & 2.89 \\
Case 2 & 2.00 & 1.15 & & \\
\hline
\end{tabular}

Table 6. Critical angles (Incident wave $P$ ).

\begin{tabular}{ccc}
$\begin{array}{c}\text { Angles critiques } \\
\text { (Degree) }\end{array}$ & $\arcsin \left(\frac{V p_{1}}{V p_{2}}\right)$ & $\arcsin \left(\frac{V p_{1}}{V s_{2}}\right)$ \\
\hline Case 1 & 44.43 & 43.79 \\
Case 2 & 23.57 & \\
\hline
\end{tabular}

Table 7. Critical angles (Incident wave S).

\begin{tabular}{ccc}
$\begin{array}{c}\text { Critical angles } \\
\text { (Degree) }\end{array}$ & $\arcsin \left(\frac{V s_{1}}{V p_{1}}\right)$ & $\arcsin \left(\frac{V s_{1}}{V p_{2}}\right)$ \\
\hline Case 1 & 35.25 & 23.87 \\
Case 2 & 35.10 & 13.30 \\
\hline
\end{tabular}
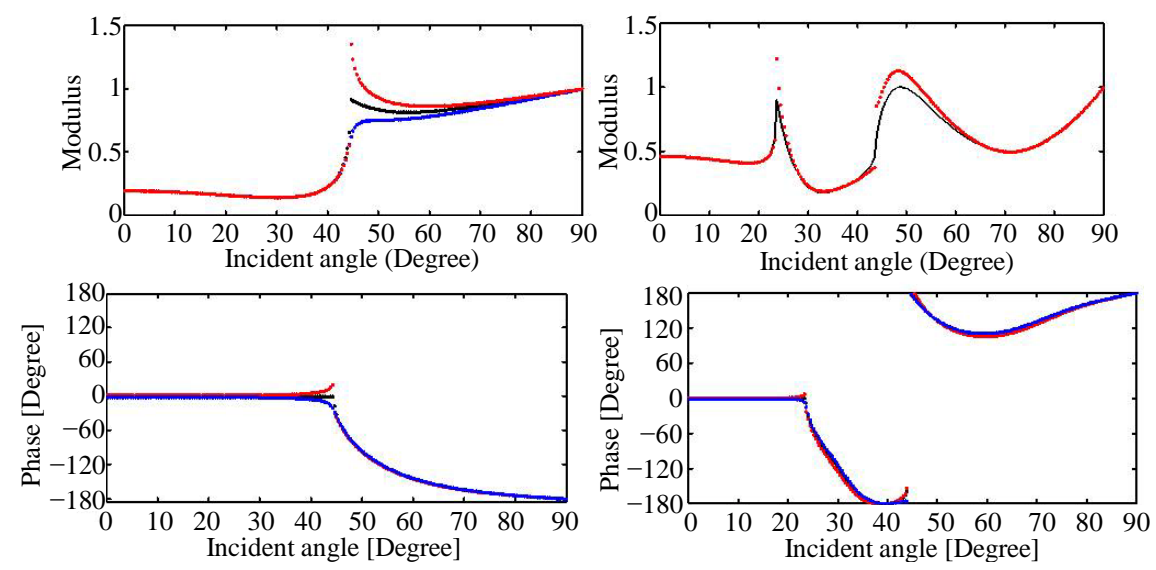

Figure 4. Modulus and phase of the reflection $R_{V}^{P \rightarrow P}$ versus the incidence angle for: $Q_{1}=20$ and $Q_{2}=1000$ (dashed red lines), $Q_{1}=1000$ and $Q_{2}=20$ (dashed blues lines), $Q_{1}=Q_{2}$ (dashed black lines). Low contrast case (left) and high contrast (right). 

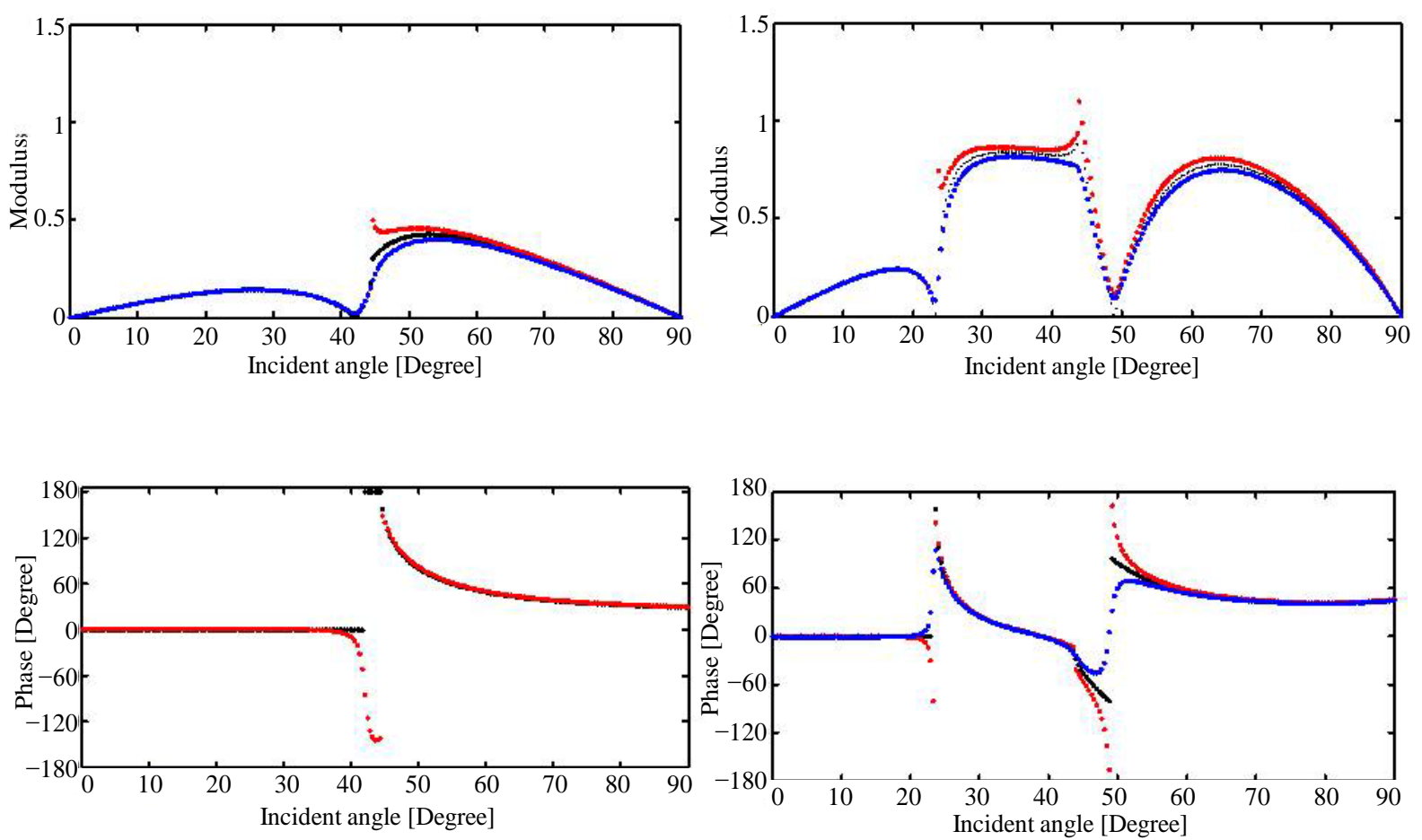

Figure 5. Modulus and phase of the reflection $R_{V}^{P \rightarrow S V}$ versus the incidence angle for: $Q_{1}=20$ and $Q_{2}=1000$ (dashed red lines), $Q_{1}=1000$ and $Q_{2}=20$ (dashed blues lines), $Q_{1}=Q_{2}$ (dashed black lines). Low contrast case (left) and high contrast (right).
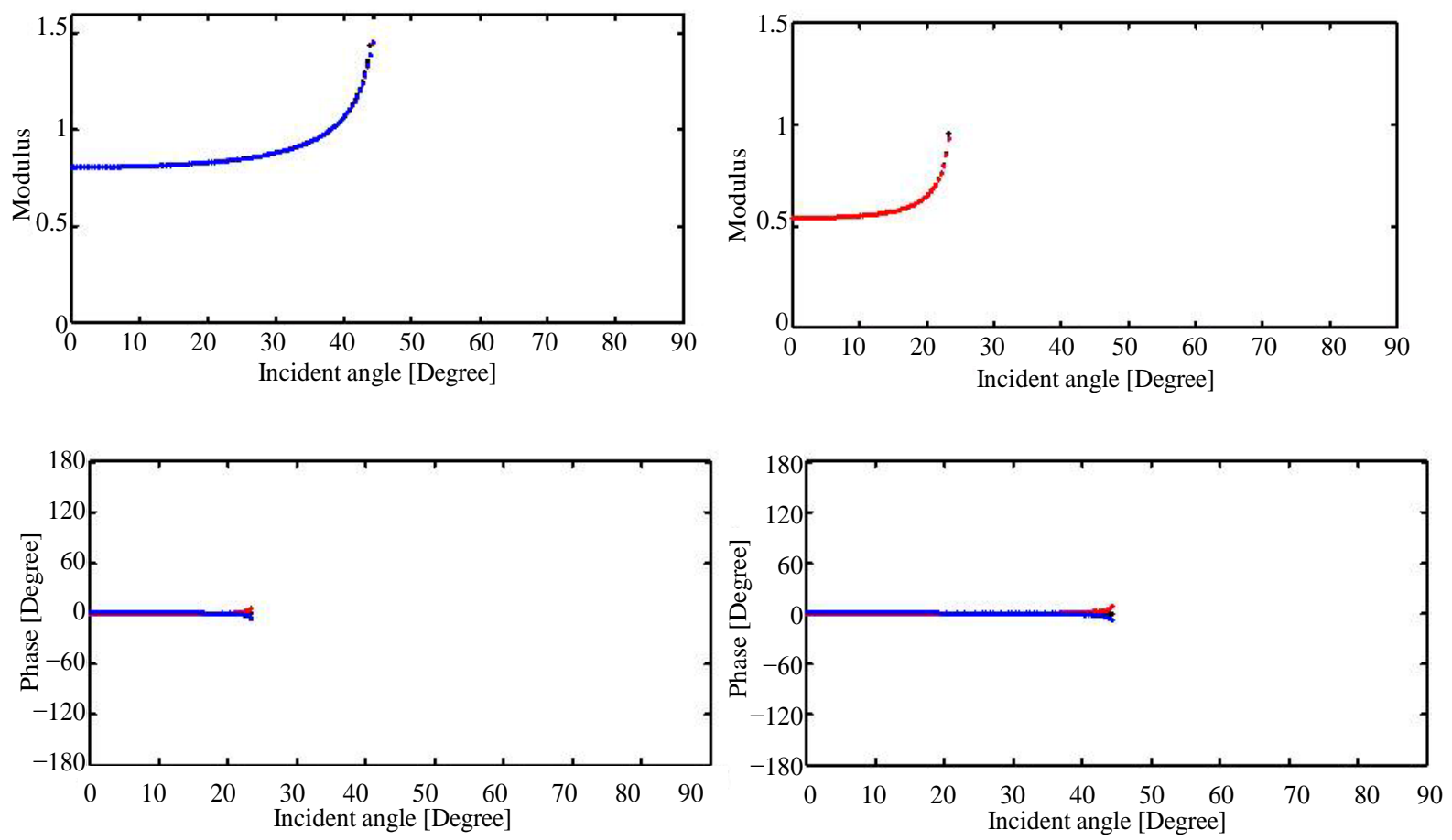

Figure 6. Modulus and phase of the transmission $T_{V}^{P \rightarrow P}$ versus the incidence angle for: $Q_{1}=20$ and $Q_{2}=1000$ (dashed red lines), $Q_{1}=1000$ and $Q_{2}=20$ (dashed blue lines), $Q_{1}=Q_{2}$ (dashed black lines). Low contrast case (left) and high contrast (right). 

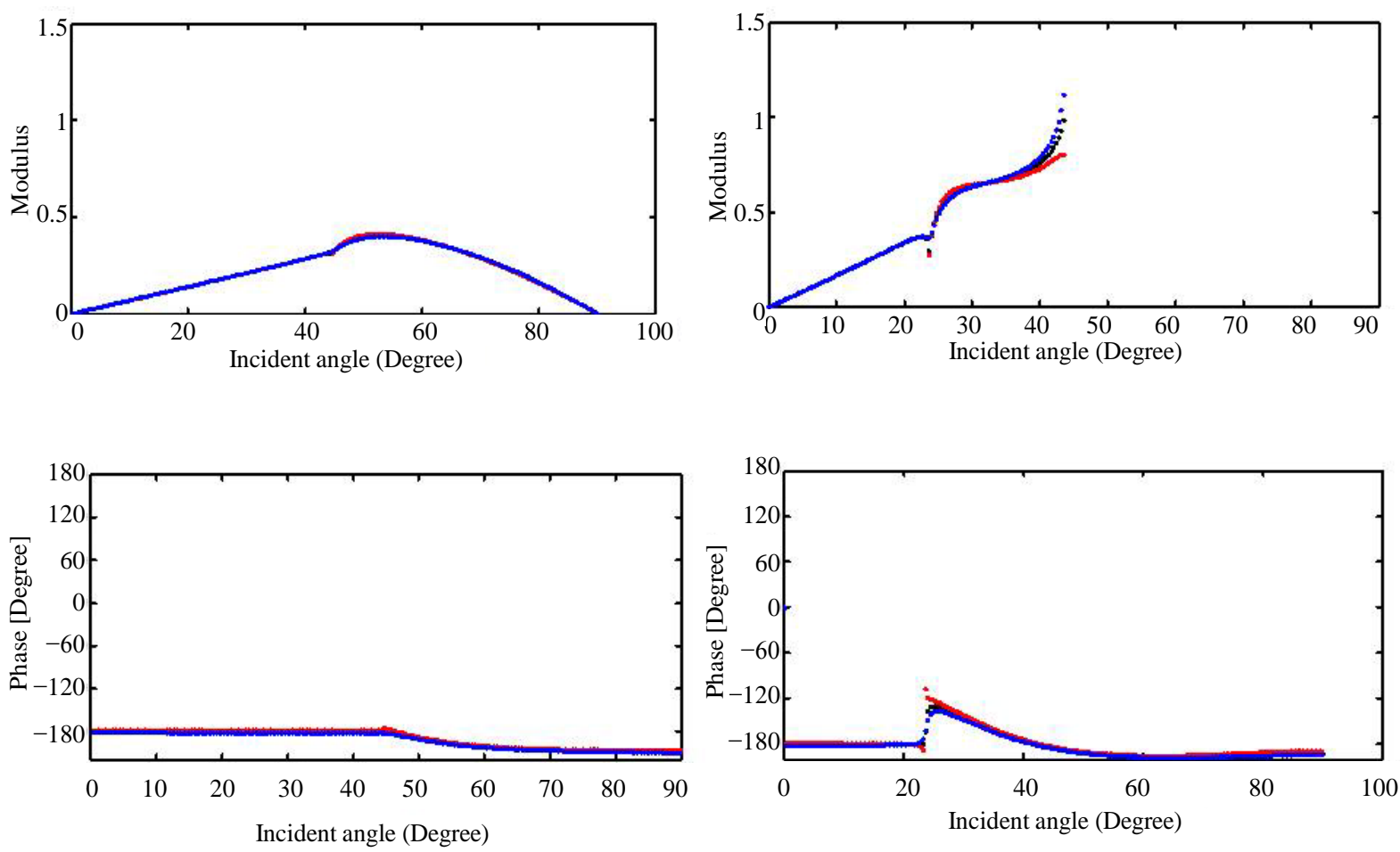

Figure 7. Modulus and phase of the transmission $T_{V}^{P \rightarrow S V}$ versus the incidence angle for: $Q_{1}=20$ and $Q_{2}=1000$ (dashed red lines), $Q_{1}=1000$ and $Q_{2}=20$ (dashed blue lines), $Q_{1}=20$ (dashed black lines). Low contrast case (left) and high contrast (right).
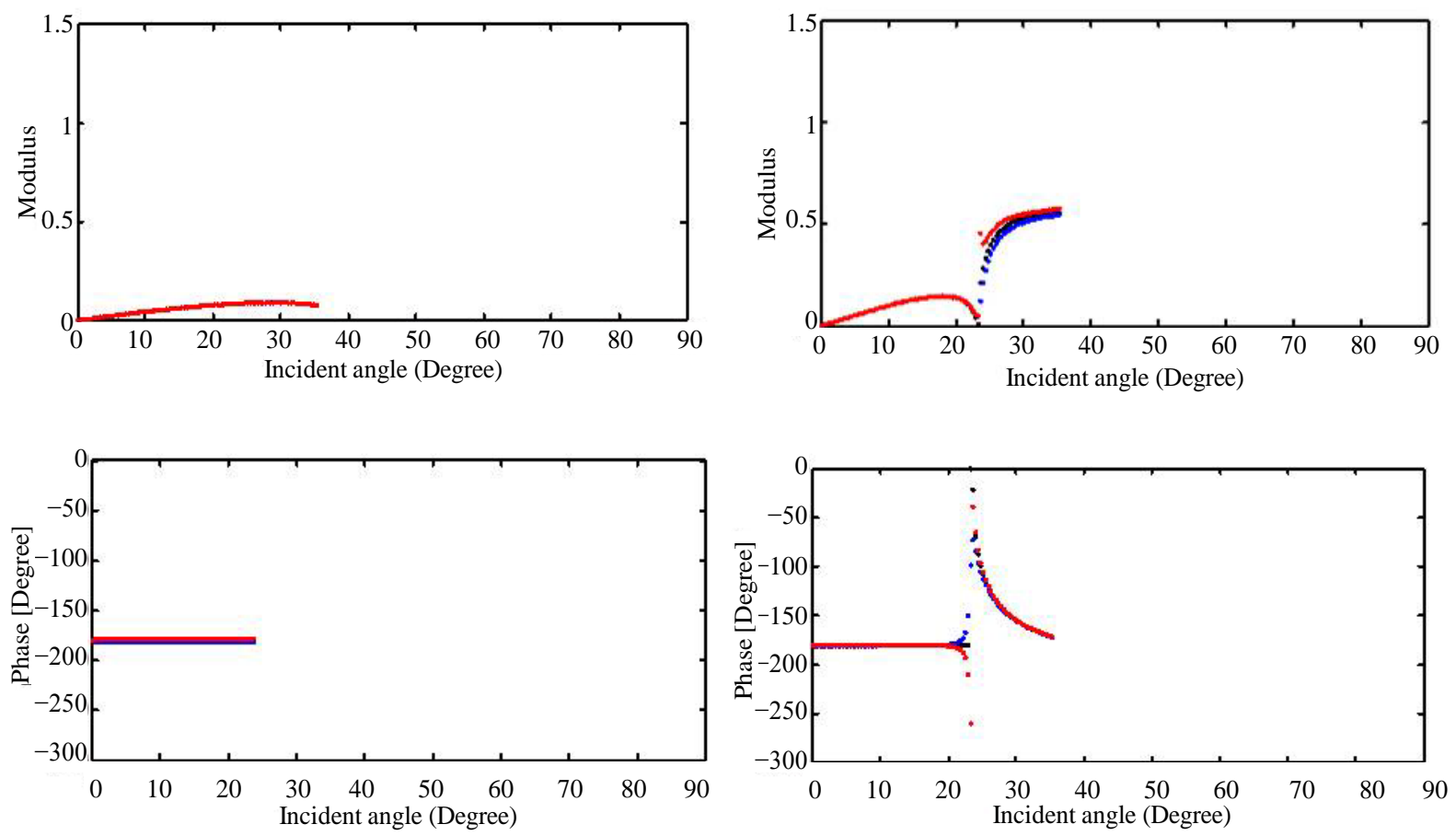

Figure 8. Modulus and phase of the transmission $R_{V}^{S V \rightarrow P}$ versus the incidence angle for: $Q_{1}=20$ and $Q_{2}=1000$ (dashed red lines), $Q_{1}=1000$ and $Q_{2}=20$ (dashed blue lines), $Q_{1}=20$ (dashed black lines). Low contrast case (left) and high contrast (right). 

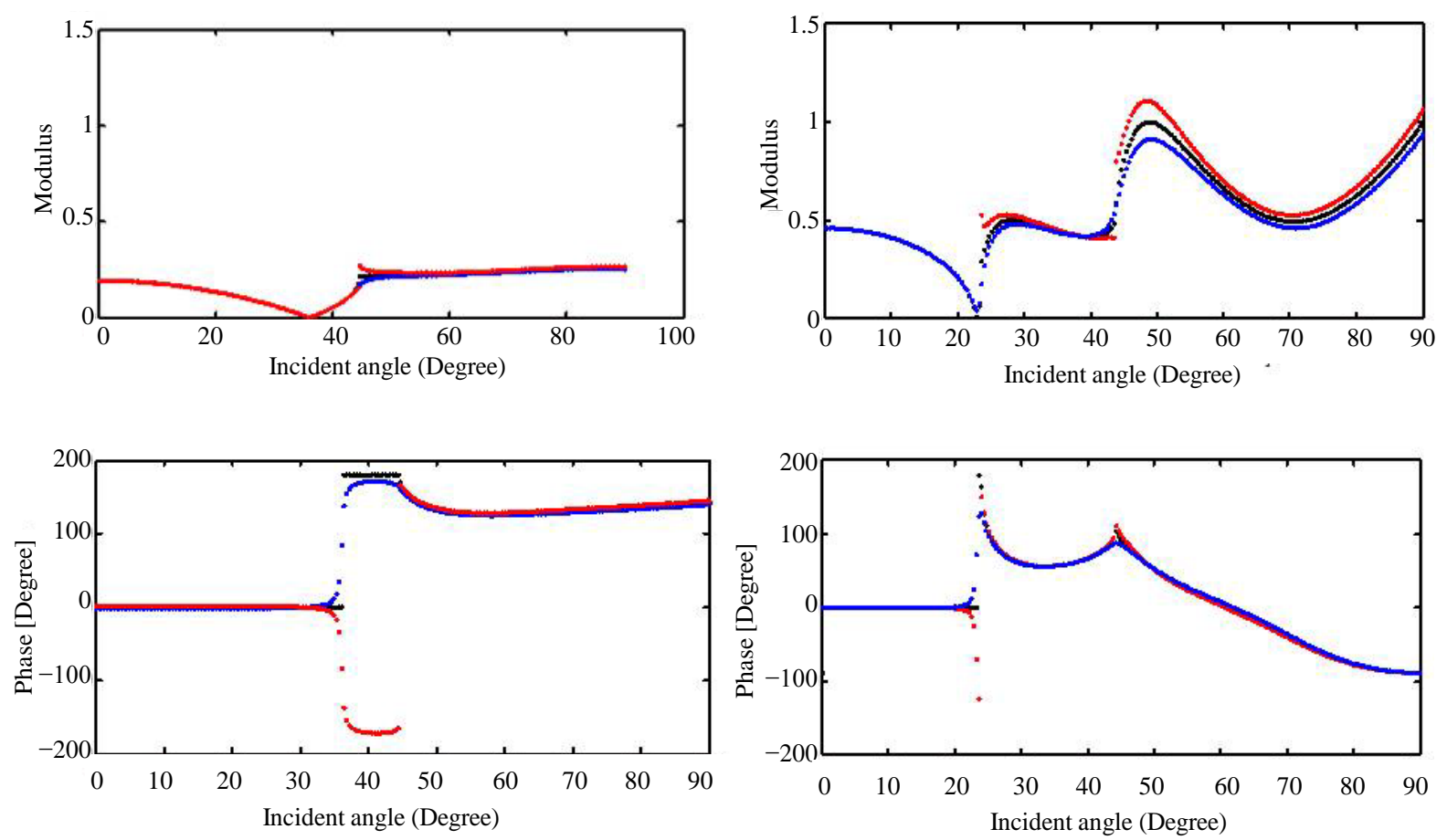

Figure 9. Modulus and phase of the transmission $R_{V}^{S V \rightarrow S V}$ versus the incidence angle for: $Q_{1}=20$ and $Q_{2}=1000$ (dashed red lines), $Q_{1}=1000$ and $Q_{2}=20$ (dashed blue lines), $Q_{1}=20$ (dashed black lines). Low contrast case (left) and high contrast (right).
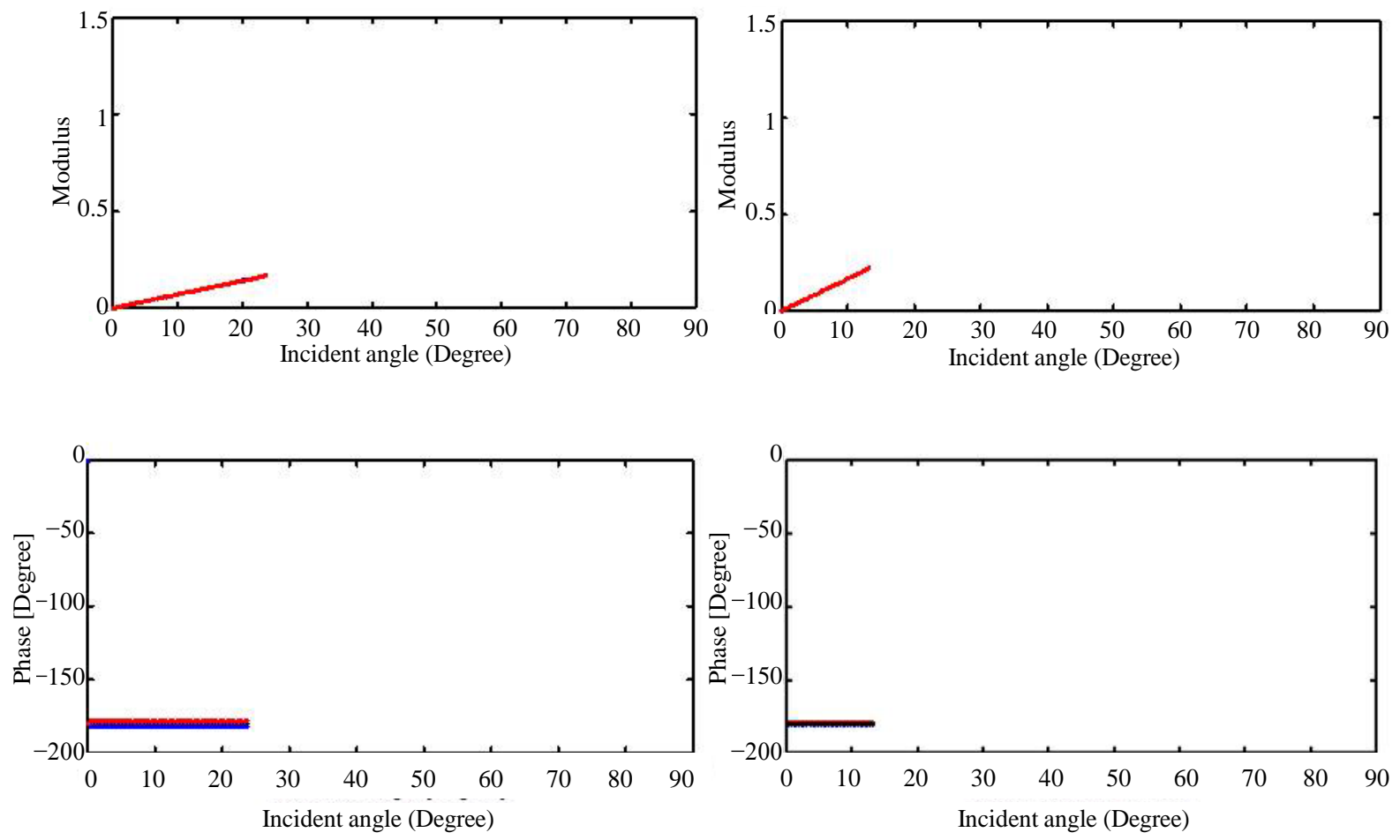

Figure 10. Modulus and phase of the transmission $T_{V}^{S V \rightarrow P}$ versus the incidence angle for: $Q_{1}=20$ and $Q_{2}=1000$ (dashed red lines), $Q_{1}=1000$ and $Q_{2}=20$ (dashed blue lines), $Q_{1}=Q_{2}$ (dashed black lines). Low contrast case (left) and high contrast (right). 

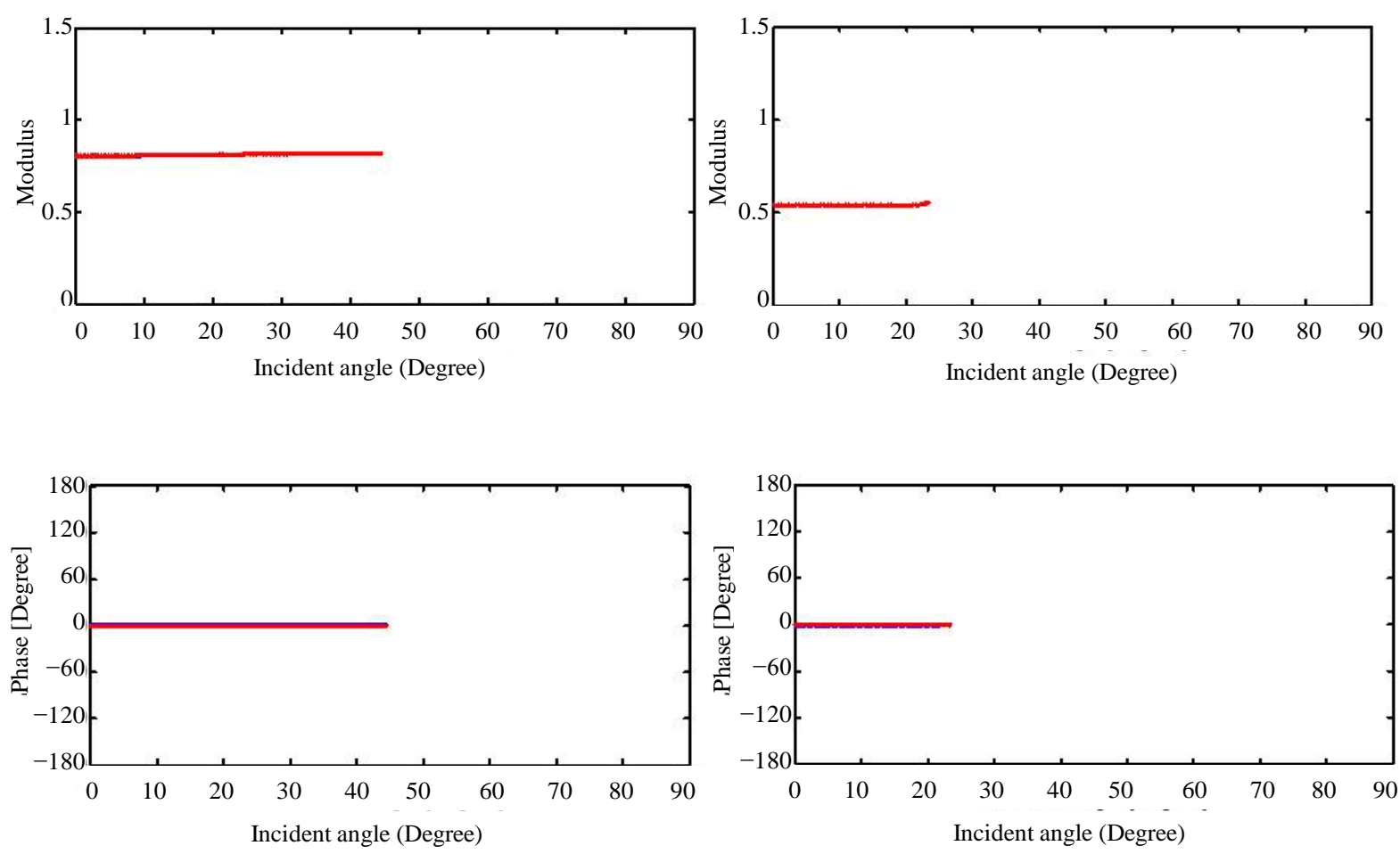

Figure 11. Modulus and phase of the transmission $T_{V}^{S V \rightarrow S V}$ versus the incidence angle for: $Q_{1}=20$ and $Q_{2}=1000$ (dashed red lines), $Q_{1}=1000$ and $Q_{2}=20$ (dashed blue lines), $Q_{1}=Q_{2}$ (dashed black lines). Low contrast case (left) and high contrast (right).
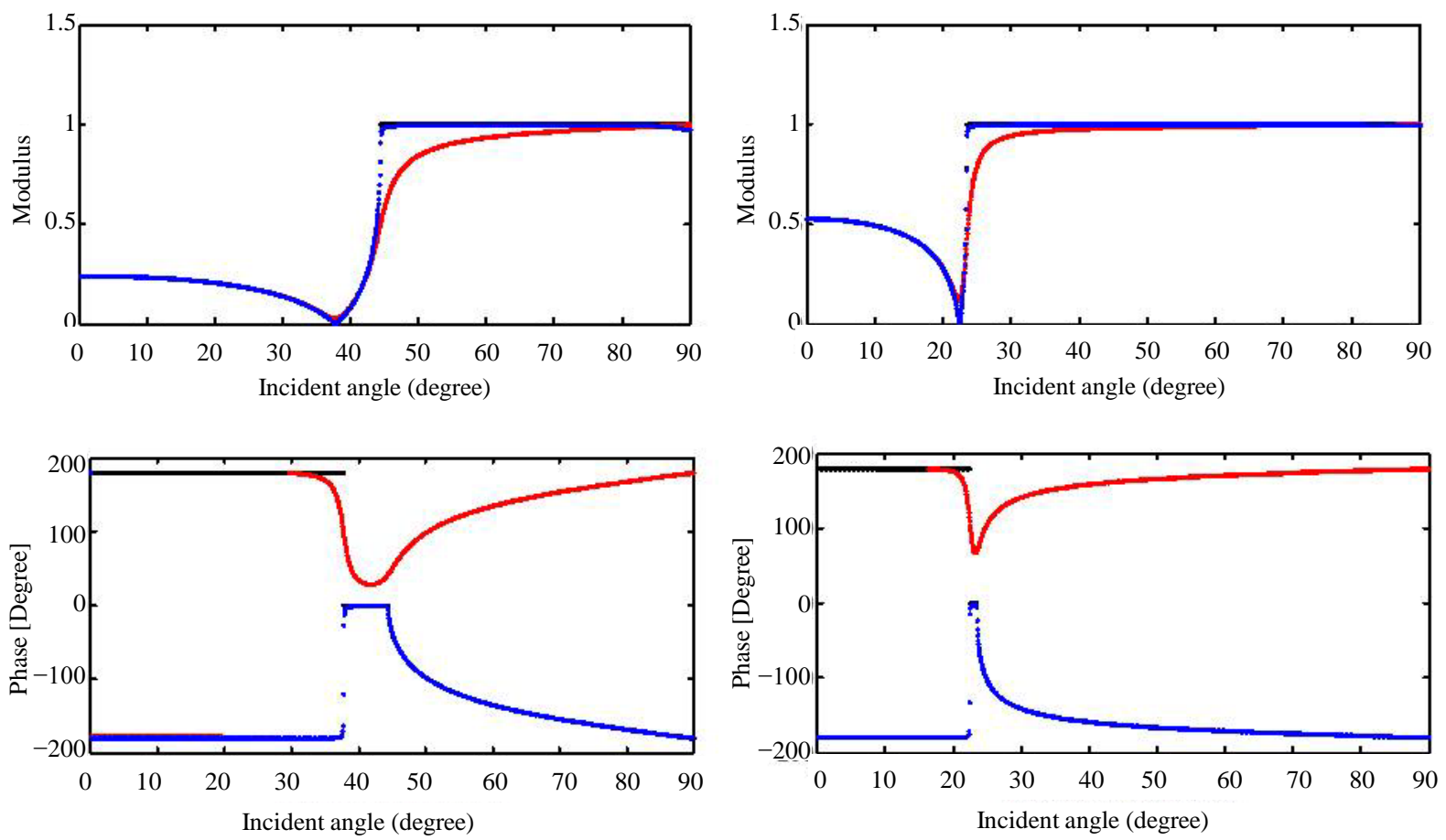

Figure 12. Modulus and phase of the transmission $R_{V}^{S H \rightarrow S H}$ versus the incidence angle for: $Q_{1}=20$ and $Q_{2}=1000$ (dashed red lines), $Q_{1}=1000$ and $Q_{2}=20$ (dashed blue lines), $Q_{1}=Q_{2}$ (dashed black lines). Low contrast case (left) and high contrast (right). 

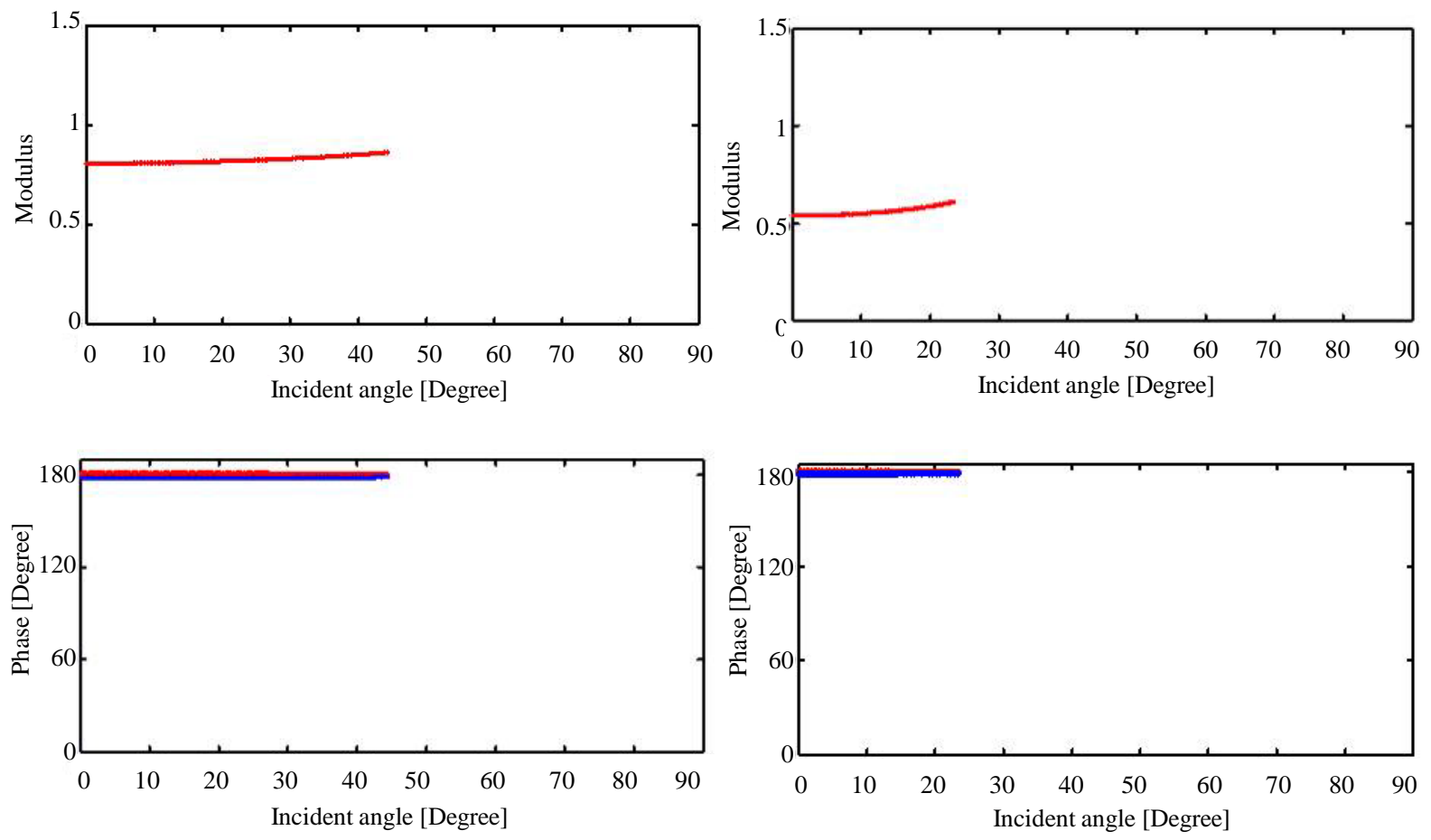

Figure 13. Modulus and phase of the transmission $T_{V}^{S H \rightarrow S H}$ versus the incidence angle for: $Q_{1}=20$ and $Q_{2}=1000$ (dashed red lines), $Q_{1}=1000$ and $Q_{2}=20$ (dashed blue lines), $Q_{1}=Q_{2}$ (dashed black lines). Low contrast case (left) and high contrast (right).

in the figures and the viscoelastic coefficients indicated by the blue and red colors can be significant. As we can note in the first case, where the modulus viscoelastic reflection coefficient around the critical angle reach 1.4, bigger than 1 because of the changing in the phase also, while in the elastic case the modulus is equal to 0.9 .

The Figures 4-13 show also that when the number of the critical angles is high, as in the case 2, the $R_{V} / T_{V}$ coefficients are more affected by the viscoelasticity of the medium.

\section{Conclusions}

The above results show that in the viscoelastic media, $R_{V} / T_{V}$ coefficients between two neighboring layers depend on the contrast of their quality factor. The effect of the contrast is effective around the critical angles, which depends on the incidence angle and the velocities of each layer. The number of the critical angles increases when the velocity contrast is large and in this case the $R_{V} / T_{V}$ coefficients are more disturbed by the viscoelasticity of the medium. We noted that when the upper layer has bigger attenuation than the lower layer $\left(Q_{1}<Q_{2}\right)$, the curves display a discontinuity at the critical angle.

The interpretation of the above observations is difficult to interpret and we do not know if it has a physical meaning or just a mathematical artefact. More understanding of the reflection/transmission mechanism in the viscoelastic media is necessary to interpret these results.

\section{References}

[1] Aki, K. and Richards, P. (1980) Quantitative Seismology. Theory and Methods. Freeman, San Francisco.

[2] Shuey, R.T. (1985) A Simplification of the Zoeppritz Equations. Geophysics, 50, 609-614. http://dx.doi.org/10.1190/1.1441936

[3] Tao, W.P., Dong, S-H. and Yang, L. (2008) Forward Modeling to Improve Seismic Reflection Energy of a Protective Coal Seam Based on Zoeppritz Equation. Geophysics, 18, 46-49.

[4] Krebes, E.S. (1983) The Viscoelastic Reflection/Transmission Problem: Two Special Cases. Bulletin of the Seismological Society of America, 73, 1673-1683. 
[5] Buchen, P.W. (1971) Reflection and Diffraction of SH-Waves in Linear Viscoelastic Solids. Geophysics, 25, 97-113.

[6] Carcione, J.M. and Tinevella, U. (2000) Bottom-Simulating Reflectors: Seismic Velocities and AVO Effects. Geophysics, 65, 54-67. http://dx.doi.org/10.1190/1.1444725

[7] Bouchaala, F. (2008) Modelisation de la propagation des ondes sismiques dans les milieuviscoelastiques: Application a la determination de l'attenuation des milieux sedimentaires. Ph.D. Dissertation, University of Western Brittany, Brest.

[8] Cerveny, V. (2001) Seismic Ray Theory. Cambridge University Press, Cambridge. http://dx.doi.org/10.1017/CBO9780511529399

[9] Lavergne, M. (1986) Methodes Sismiques. Technip, Paris. 
Appendix

$$
\begin{aligned}
& \boldsymbol{p}_{i} \cdot \boldsymbol{X}_{1}=\boldsymbol{p}_{r} \cdot \boldsymbol{X}_{1}=\boldsymbol{p}_{t} \cdot \boldsymbol{X}_{1}=\|\boldsymbol{a}\|=a \\
& \boldsymbol{p}_{i}^{*} \cdot \boldsymbol{X}_{2}=\boldsymbol{p}_{r}^{*} \cdot \boldsymbol{X}_{2}=\boldsymbol{p}_{t}^{*} \cdot \boldsymbol{X}_{2}=0 \\
& \boldsymbol{p}_{i}^{*} \cdot \boldsymbol{X}_{3}=-\frac{1}{V_{i}^{*}}\left(1-a^{* 2} V_{i}^{* 2}\right)^{1 / 2} \\
& \boldsymbol{p}_{r}^{*} \cdot \boldsymbol{X}_{3}=\frac{1}{V_{r}^{*}}\left(1-a^{* 2} V_{r}^{* 2}\right)^{1 / 2} \\
& \boldsymbol{p}_{t}^{*} \cdot \boldsymbol{X}_{3}=-\frac{1}{V_{t}^{*}}\left(1-a^{* 2} V_{t}^{* 2}\right)^{1 / 2}
\end{aligned}
$$

Expression of the reflection-transmission coefficients $(P-S V)$ in isotropic, inhomogeneous and viscoelastic medium

$$
\begin{aligned}
& R_{V}^{P \rightarrow P}=\left(A^{*}+B^{*}-C^{*}-D^{*}+E^{*}-F^{*}\right) \tilde{D}^{*-1}, \\
& R_{V}^{P \rightarrow S V}=2 p^{*} \alpha_{1}^{*} P_{1}^{*}\left(q^{*} P_{3}^{*} P_{4}^{*} Y^{*}+\alpha_{2}^{*} \beta_{2}^{*} X^{*} Z^{*}\right) \tilde{D}^{*-1}, \\
& T_{V}^{P \rightarrow P}=2 \rho_{1} \alpha_{1}^{*} P_{1}^{*}\left(\beta_{2}^{*} P_{2}^{*} X^{*}+\beta_{1}^{*} P_{4}^{*} Y^{*}\right) \tilde{D}^{*-1}, \\
& T_{V}^{P \rightarrow S V}=-2 \rho_{1} p^{*} \alpha_{1}^{*} P_{1}^{*}\left(q^{*} P_{2}^{*} P_{3}^{*}-\beta_{1}^{*} \alpha_{2}^{*} Z^{*}\right) \tilde{D}^{*-1}, \\
& R_{V}^{S V \rightarrow S V}=\left(A^{*}-B^{*}+C^{*}-D^{*}+E^{*}-F^{*}\right) \tilde{D}^{*-1}, \\
& R_{V}^{S V \rightarrow P}=-2 p^{*} \beta_{1}^{*} P_{2}^{*}\left(q^{*} P_{3}^{*} P_{4}^{*} Y^{*}+\alpha_{2}^{*} \beta_{2}^{*} X^{*} Z^{*}\right) \tilde{D}^{*-1}, \\
& T_{V}^{S V \rightarrow S V}=2 \rho_{1} \beta_{1}^{*} P_{2}^{*}\left(\alpha_{1}^{*} P_{3}^{*} Y^{*}+\alpha_{2}^{*} P_{1}^{*} X^{*}\right) \tilde{D}^{*-1}, \\
& T_{V}^{S V \rightarrow P}=2 \rho_{1} p^{*} \beta_{1}^{*} P_{2}^{*}\left(q^{*} P_{1}^{*} P_{4}^{*} Y^{*}-\alpha_{1}^{*} \beta_{2}^{*} Z^{*}\right) \tilde{D}^{*-1} .
\end{aligned}
$$

with: $\tilde{D}^{*}=A^{*}+B^{*}+C^{*}+D^{*}+E^{*}+F^{*}$

$$
\begin{aligned}
& A^{*}=p^{* 2} q^{* 2} P_{1}^{*} P_{2}^{*} P_{3}^{*} P_{4}^{*}, \quad B^{*}=\rho_{1} \rho_{2} \beta_{1}^{*} \alpha_{2}^{*} P_{1}^{*} P_{4}^{*}, \quad C^{*}=\rho_{1} \rho_{2} \alpha_{1}^{*} \beta_{2}^{*} P_{2}^{*} P_{3}^{*}, \\
& D^{*}=\alpha_{1}^{*} \beta_{1}^{*} P_{3}^{*} P_{4}^{*} Y^{* 2}, \quad E^{*}=\alpha_{2}^{*} \beta_{2}^{*} P_{1}^{*} P_{2}^{*} X^{* 2}, \quad F^{*}=p^{* 2} \alpha_{1}^{*} \alpha_{2}^{*} \beta_{1}^{*} \beta_{2}^{*} Z^{* 2} .
\end{aligned}
$$

and $X^{*}=\rho_{2}-q^{*} p^{* 2} \quad Y^{*}=\rho_{1}+q^{*} p^{* 2} \quad Z^{*}=\rho_{2}-\rho_{1}-q^{*} p^{* 2}$

$$
q^{*}=2\left(\rho_{2} \beta_{2}^{* 2}-\rho_{1} \beta_{1}^{* 2}\right)
$$

After doing the below substitution in the above expressions of reflection-transmission coefficients,

$$
\begin{gathered}
\alpha_{i}^{*}=\alpha_{i}\left(1-\frac{i}{2 Q_{i}}\right) \quad \beta_{i}^{*}=\beta_{i}\left(1-\frac{i}{2 Q_{i}}\right) \text { for } i=1,2 \\
p^{*}=p_{e}\left(1+\frac{i}{2 Q_{1}}\right)
\end{gathered}
$$

and $p_{e}=\frac{\sin \left(\theta_{i}\right)}{\alpha_{1}}$ for $P$ incident wave and $p_{e}=\frac{\sin \left(\theta_{i}\right)}{\beta_{1}}$ for $S$ incident wave

we obtain:

$$
\begin{aligned}
& q^{*}=2\left(\rho_{2} \beta_{2}^{2}-\rho_{1} \beta_{1}^{2}\right)-2 i\left(\frac{\rho_{2} \beta_{2}^{2}}{Q_{2}}-\frac{\rho_{1} \beta_{1}^{2}}{Q_{1}}\right) \\
& X^{*}=\rho_{2}-2\left(\rho_{2} \beta_{2}^{2}-\rho_{1} \beta_{1}^{2}\right) p_{e}^{2}+2 i p_{e}^{2} \rho_{2} \beta_{2}^{2}\left(\frac{1}{Q_{2}}-\frac{1}{Q_{1}}\right),
\end{aligned}
$$




$$
\begin{aligned}
& Y^{*}=\rho_{1}+2\left(\rho_{2} \beta_{2}^{2}-\rho_{1} \beta_{1}^{2}\right) p_{e}^{2}-2 i p_{e}^{2} \rho_{2} \beta_{2}^{2}\left(\frac{1}{Q_{2}}-\frac{1}{Q_{1}}\right) \\
& Z^{*}=\rho_{2}-\rho_{1}-2\left(\rho_{2} \beta_{2}^{2}-\rho_{1} \beta_{1}^{2}\right) p_{e}^{2}+2 i p_{e}^{2} \rho_{2} \beta_{2}^{2}\left(\frac{1}{Q_{2}}-\frac{1}{Q_{1}}\right) .
\end{aligned}
$$

And also,

$$
\begin{aligned}
& P_{1}^{*}=\left(1-p_{e}^{2} \alpha_{1}^{2}\right)^{\frac{1}{2}}, \\
& P_{2}^{*}=\left(1-p_{e}^{2} \beta_{1}^{2}\right)^{\frac{1}{2}}, \\
& P_{3}^{*}=\left(1-p_{e}^{2} \alpha_{2}^{2}\right)^{\frac{1}{2}}\left(1+i \frac{\alpha_{2}^{2} p_{e}^{2}}{2\left(1-p_{e}^{2} \alpha_{2}^{2}\right)}\left(\frac{1}{Q_{2}}-\frac{1}{Q_{1}}\right)\right), \\
& P_{4}^{*}=\left(1-p_{e} \beta_{2}^{2}\right)^{\frac{1}{2}}\left(1+i \frac{\beta_{2}^{2} p^{2}}{2\left(1-p_{e}^{2} \beta_{2}^{2}\right)}\left(\frac{1}{Q_{2}}-\frac{1}{Q_{1}}\right)\right) .
\end{aligned}
$$

Now we compute $\tilde{D}^{*}$ and $\tilde{D}^{*-1}$

$$
\begin{aligned}
& \tilde{D}^{*}=\tilde{D}+i(a A+b B+c C+d D+e E+f F), \\
& \tilde{D}^{*-1}=\tilde{D}^{-1}-\frac{i}{\tilde{D}^{2}}(a A+b B+c C+d D+e E+f F) .
\end{aligned}
$$

With $\tilde{D}=A+B+C+D+E+F$

$$
\begin{aligned}
& A=4 p_{e}^{2}\left(\rho_{2} \beta_{2}^{2}-\rho_{1} \beta_{1}^{2}\right)^{2}\left(1-p_{e}^{2} \alpha_{1}^{2}\right)^{\frac{1}{2}}\left(1-p_{e}^{2} \beta_{1}^{2}\right)^{\frac{1}{2}}\left(1-p_{e}^{2} \alpha_{2}^{2}\right)^{\frac{1}{2}}\left(1-p_{e}^{2} \beta_{2}^{2}\right)^{\frac{1}{2}}, \\
& B=\rho_{1} \rho_{2} \beta_{1} \alpha_{2}\left(1-p_{e}^{2} \alpha_{1}^{2}\right)^{\frac{1}{2}}\left(1-p_{e}^{2} \beta_{2}^{2}\right)^{\frac{1}{2}}, \\
& C=\rho_{1} \rho_{2} \alpha_{1} \beta_{2}\left(1-p_{e}^{2} \beta_{1}^{2}\right)^{\frac{1}{2}}\left(1-p_{e}^{2} \alpha_{2}^{2}\right)^{\frac{1}{2}}, \\
& D=\alpha_{1} \beta_{1}\left(1-p_{e}^{2} \alpha_{2}^{2}\right)^{\frac{1}{2}}\left(1-p_{e}^{2} \beta_{2}^{2}\right)^{\frac{1}{2}}\left[\rho_{1}+2\left(\rho_{2} \beta_{2}^{2}-\rho_{1} \beta_{1}^{2}\right) p_{e}^{2}\right], \\
& E=\alpha_{2} \beta_{2}\left(1-p_{e}^{2} \alpha_{1}^{2}\right)^{\frac{1}{2}}\left(1-p_{e}^{2} \beta_{1}^{2}\right)^{\frac{1}{2}}\left[\rho_{2}-2\left(\rho_{2} \beta_{2}^{2}-\rho_{1} \beta_{1}^{2}\right) p_{e}^{2}\right], \\
& F=p_{e}^{2} \alpha_{1} \alpha_{2} \beta_{1} \beta_{2}\left[\rho_{2}-\rho_{1}-2\left(\rho_{2} \beta_{2}^{2}-\rho_{1} \beta_{1}^{2}\right) p_{e}^{2}\right] .
\end{aligned}
$$

Table A1. Component in the basis $\left(\boldsymbol{X}_{1}, \boldsymbol{X}_{2}, \boldsymbol{X}_{3}\right)$ of stress vector at $I$ due to incident, reflected, and transmitted waves (Reflection/Transmission $P$-SV).

$$
\begin{array}{rlrl}
A^{*} & =A(1-i a) & a=\frac{4}{q}\left(\frac{\rho_{2} \beta_{2}^{2}}{Q_{2}}-\frac{\rho_{1} \beta_{1}^{2}}{Q_{1}}\right)-\frac{1}{Q_{1}}-\left(\frac{\alpha_{2}^{2} p^{2}}{2 P_{3}^{2}}+\frac{\beta_{2}^{2} p^{2}}{2 P_{4}^{2}}\right)\left(\frac{1}{Q_{2}}-\frac{1}{Q_{1}}\right) \\
B^{*} & =B(1-i b) & b=\frac{1}{2 Q_{1}}+\frac{1}{2 Q_{2}}-\frac{\beta_{2}^{2} p^{2}}{2 P_{4}^{2}}\left(\frac{1}{Q_{2}}-\frac{1}{Q_{1}}\right) \\
C^{*}=C(1-i c) & c=\frac{1}{2 Q_{1}}+\frac{1}{2 Q_{2}}-\frac{\alpha_{2}^{2} p^{2}}{2 P_{3}^{2}}\left(\frac{1}{Q_{2}}-\frac{1}{Q_{1}}\right) \\
D^{*}=D(1-i d) & d=\frac{1}{Q_{1}}-\left(\frac{\alpha_{2}^{2} p^{2}}{2 P_{3}^{2}}+\frac{\beta_{2}^{2} p^{2}}{2 P_{4}^{2}}\right)\left(\frac{1}{Q_{2}}-\frac{1}{Q_{1}}\right)+4 \frac{p^{2} \rho_{2} \beta_{2}^{2}}{Y}\left(\frac{1}{Q_{2}}-\frac{1}{Q_{1}}\right) \\
E^{*}=E(1-i e) & e=\frac{1}{Q_{2}}-4 \frac{p^{2} \rho_{2} \beta_{2}^{2}}{X}\left(\frac{1}{Q_{2}}-\frac{1}{Q_{1}}\right) \\
F^{*}=F(1-i f) & f=\frac{1}{Q_{2}}-4 \frac{p^{2} \rho_{2} \beta_{2}^{2}}{Z}\left(\frac{1}{Q_{2}}-\frac{1}{Q_{1}}\right)
\end{array}
$$


Scientific Research Publishing (SCIRP) is one of the largest Open Access journal publishers. It is currently publishing more than 200 open access, online, peer-reviewed journals covering a wide range of academic disciplines. SCIRP serves the worldwide academic communities and contributes to the progress and application of science with its publication.

Other selected journals from SCIRP are listed as below. Submit your manuscript to us via either submit@scirp.org or Online Submission Portal.
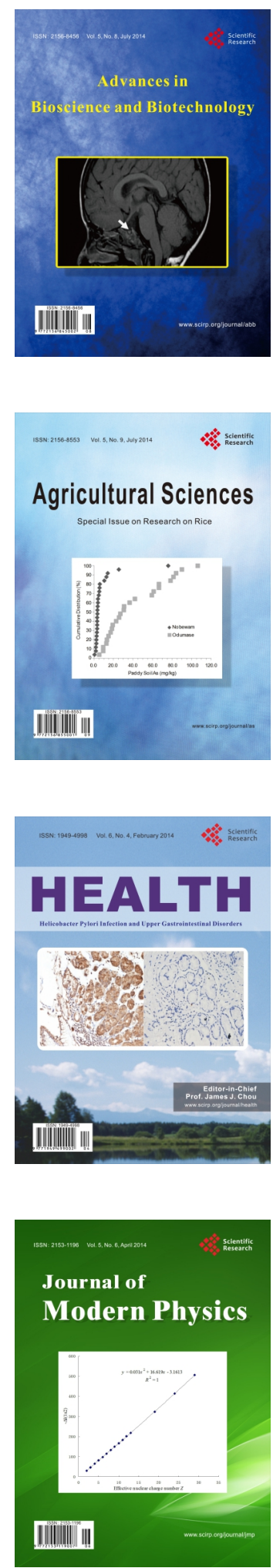
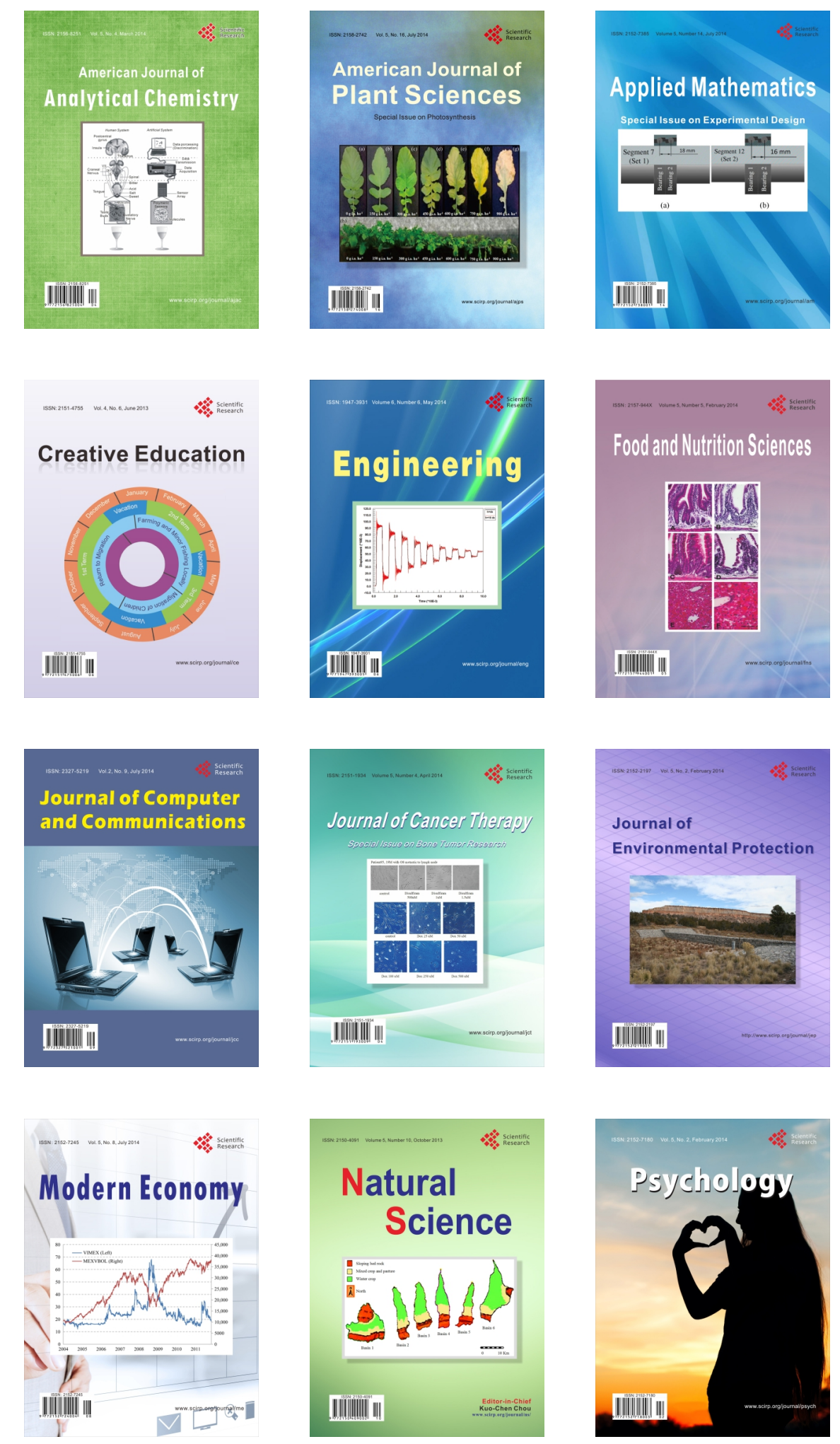